\title{
Memory Encoding Processes in Young and Old Adults
}

\author{
Michael C. C. Kuo ${ }^{1}$; Karen P. Y. Liu ${ }^{2, *}$; Michelle Bissett ${ }^{2}$; Jacqueline Wesson ${ }^{3,4}$; Nikki Tulliani ${ }^{2}$; \\ Rosalind $\mathrm{BYE}^{2}$; Leung-Wing $\mathrm{Chu}^{5,6}$ \\ ${ }_{1}^{1}$ Department of Applied Sciences, Hong Kong Institute of Vocational Education, Hong Kong, China \\ 2 University of Western Sydney, Sydney, Australia \\ ${ }^{3}$ Ageing Work and Health Research Unit, University of Sydney, Sydney, Australia \\ ${ }_{4}^{4}$ Ageing Work and Health Research Unit, University of Sydney, Sydney, Australia \\ 5 Academic Department of Aged Care Psychiatry, Prince of Wales Hosp \\ 6 Alzheimer's Disease Research Network, The University of Hong Kong, Hong Kong \\ *Corresponding author: Karen P. Y. Liu, University of Western Sydney, Sydney Australia. Tel:+61-246203432, Fax:+61-246203792, E-mail: Karen.Liu@uws.edu.au
}

Received: Feb 30, 2014; Revised: Mar 15, 2014; Accepted: Mar 30, 2014

\begin{abstract}
Context: Information processing requires a series of mental operations from encoding to storage and retrieval. A large number of studies have examined the retrieval process, but less attention has been paid to the encoding process.

Evidence Acquisition: The aims of this paper were to provide an overview of perceptual and semantic encoding processes as well as identify and compare the structural and functional changes in the memory encoding process of young and old adults. A review of encoding was conducted based on findings from two recent memory encoding studies and a literature search on memory encoding. It included studies published from January 1980 to December 2013 and appeared in the databases of CINAHL, MEDLINE, AMED, Journals@ Ovid and the ISI Web of Science.

Results: Five stages of encoding were identified. They were:1) early perceptual processing; 2 ) prelexical feature detection and early lexical processing; 3) initial selection of information from competing information for processing in the working memory; 4) semantic, syntactic, or lexical processing; and 5) updating of working memory with the newly created item representation and elaborate processing. Perceptual encoding appears to be modulated by sensory cortices, in which stimuli are initially perceived and processed, whereas semantic encoding involves the left prefrontal cortex temporal regions by both verbal and nonverbal stimuli.

Conclusions: Early perceptual processing and the selection of information processes associated with memory encoding are comparable between young and old adults. However, they showed differences in semantic processing, updating of working memory, and elaborate processing. These differences might be due to reduced volume and functional capacity of the hippocampus and frontal lobes, shrinkage in the white matter and its associated tracts, as well as inability of the aging brain to produce levels of dopamine comparable to its previous levels.
\end{abstract}

Keywords:Memory; Perceptual; Semantic; Adults

\section{Context}

The model of information processing envisions memory as a series of mental operations from encoding to storage, then to retrieval (1). According to this view, information is perceived, placed in short-term memory, and then processed in working memory. Eventually, it is consolidated into long-term memory and retrieved upon request. Encoding and retrieval are closely related. A large number of studies have examined the retrieval process. The encoding process is comparatively less researched, possibly because encoding can only be measured by some form of test. These can be either an implicit test such as word fragment completion or an explicit test such as free-recall, at retrieval (2). Encoding has been defined as 'the set of processes involved in transforming external events and internal thoughts into both temporary and long-lasting neural representations' (2). Although the cognitive processes involved in encoding are not entirely clear, research techniques, such as those investigating the brain electrical activities have been used to investigate these processes. Paying attention to the item, transferring and processing the selected ideas in the working memory, building connections between selected ideas to create a mental model, and creating links between these new models and prior knowledge in long-term memory have been the basic stages theorized to be involved in encoding $(3,4)$.

Information can be processed perceptually or semantically (5). Encoding strategies can therefore be classicould also provide possible intervention strategies for older adults with mild cognitive impairments. 
fied as either perceptual or semantic $(6,7)$. The chunking theory (8) is one of the well-known strategies which refers to dividing the information into different meaning groups during encoding to facilitate their retrieval. Pathways of dividing the information into groups include focusing on the shapes and categories of the objects to be encoded. Encoding objects by shape, based on the details of our perception, is referred as a perceptual encoding strategy. On the other hand, encoding objects by categories, based on their implicit meaning, is known as semantic encoding strategy.

\section{Evidence Acquisition}

This purpose of this paper was to provide a brief overview of perceptual and semantic encoding processes in young and old adults based on our two previous studies $(7,9)$ and discuss the age-related structural and functional changes by reviewing the related literature. A literature search on memory encoding was completed to further support the encoding process identified. Reports of researches published from January 1980 to December 2013, available in the databases of CINAHL (1982 to date), MEDLINE (1966 to date), AMED (1985 to date), Journals@Ovid, and the ISI Web of Science (1900 to date for both) were collected and reviewed.

\section{Results}

\subsection{Summary of Perceptual and Semantic Encod- ing Studies}

In our two previous studies, Kuo and Liu (7) investigated the use of the two encoding strategies in healthy young adults (mean age $=21.5$ ) and Kuo et al. (9) compared the use of the same encoding strategies for healthy young (mean age $=21.8$ ) and old (mean age $=$ $65.5)$ adults. All participants underwent a memory encoding task experiment, consisting of two parts. Firstly, participants studied a series of Chinese characters either perceptually (by inspecting orthographic components) (known as perceptual condition) or semantically (by determining the object making sounds) (known as semantic condition). Allocation to either perceptual or semantic condition was randomized across the participants. In the second part, a selection of Chinese characters were shown to the participants and they must judge whether the viewed items had been studied.

A total of 402 common Chinese characters were selected and divided to four 40-item encoding lists (part 1) and eight 40-item recognition lists (part 2). Each of the eight recognition blocks was constructed by randomly mixing 20 items from the encoding lists and 20 items from the unused characters. These unused characters had similar complexity to the studied characters. The character stimuli were prepared in black against a white background with a font size of 140 using the STIM2 (Stimulus presentation and experimental design system) software (Compumedics Neuroscan, Charlotte, North Carolina, USA) on a 17-inch Cathode Ray Tube monitor. During the recognition phase, participants responded by determining whether the stimulus had been studied in the study block, with equal emphasis on speed and accuracy. The results were recorded using the following responses: correctly identified (studied item that was correctly identified as studied), missed (studied item that was not correctly identified as studied), false alarm (unstudied item that was incorrectly identified as studied) and correctly rejected (unstudied item that was correctly identified as unstudied).

For the young adults group in both studies $(7,9)$, the fixation cross at the study and recognition phases was presented for $500 \mathrm{~ms}$, followed by a character stimulus for $400 \mathrm{~ms}$. The response time allowed at the study phase was $3500 \mathrm{~ms}$, and the time allowed at the recognition phase was $2500 \mathrm{~ms}$. The total time a younger participant spent completing the blocks in either perceptual or semantic condition was 13 minutes and 20 seconds. For the older group in Kuo and Liu's study (9), timing of the paradigm was similar to that used for the younger group with two exceptions; the fixation cross at the study and recognition phases was presented for $1000 \mathrm{~ms}$ instead of $500 \mathrm{~ms}$ as in the younger group, and the response time allowed at the recognition phase was $3500 \mathrm{~ms}$. The total average time an older participant spent completing all of the study and recognition blocks in either perceptual or semantic condition was approximately 16 minutes.

Subsequent memory effect (SME) has been used to reflect the processes of encoding $(10,11)$. It is a common event-related potential(ERP) index that reflects processes during encoding (11). It is defined as the differences in amplitude between the ERPs elicited during encoding for subsequently successfully retrieved trials versus the missed trials for example. $(7,10,12)$. Electroencephalograms (EEGs) were recorded during the encoding phase using the Quikcap system of the SynAmps2 amplifier and Acquire 4.3 software (Compumedics Neuroscan, Charlotte, North Carolina, USA). The averaged ERPs were computed per participant by sorting epochs that were subsequently correctly identified or missed during the recognition phase. The stages of encoding were identified based on the results of the peaks generated in the ERPs.

Results of Kuo and Liu's study (7) showed that the younger adults had similar accuracy levels in identifying the studied characters in semantic and perceptual conditions ( $1.94 \pm 0.57$ vs. $1.79 \pm 0.6)$ and no difference in reaction times in recognition. In both conditions, the SMEs were more positive-going waveforms for the correctly identified trials than missed trials and significant in windows between 120 and $240 \mathrm{~ms}$ (P2), 240 and $360 \mathrm{~ms}$ (N3), 360 and $700 \mathrm{~ms}$ (P550), and 700 and $1000 \mathrm{~ms}$ (late positive component; LPC) (Figure 1). SME of P2 has been suggested to be related to working memory or attentional operations, SME of N3 to conceptual processing, and SME 
Figure 1. Subtracted Waveforms of the Event-Related Potentials Elicited by Correctly Identified and Missed Trials

A

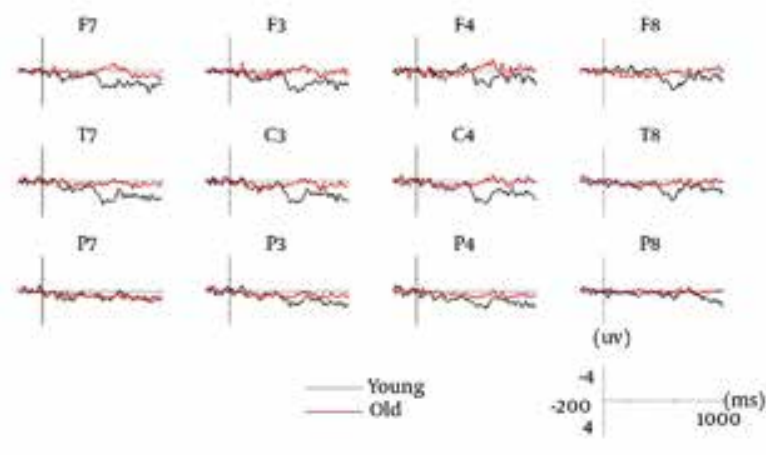

B

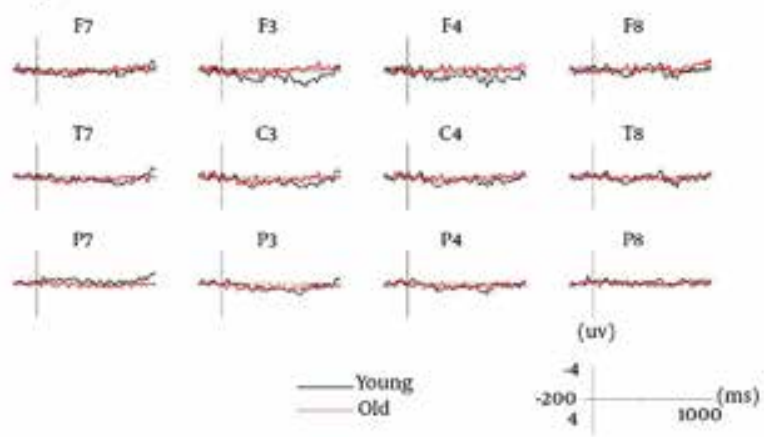

(A) perceptual condition and (B) semantic condition of the younger and older groups (9). A selection of 12 electrode locations from left (F3, C3, P3, F7, T7, P7) and right (F4, C6, P4, F8, T8, P8) hemispheres are shown.

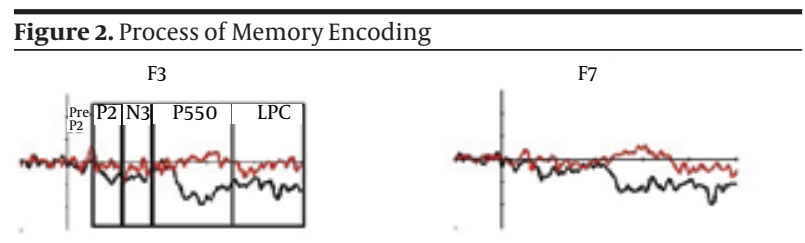

(uv)

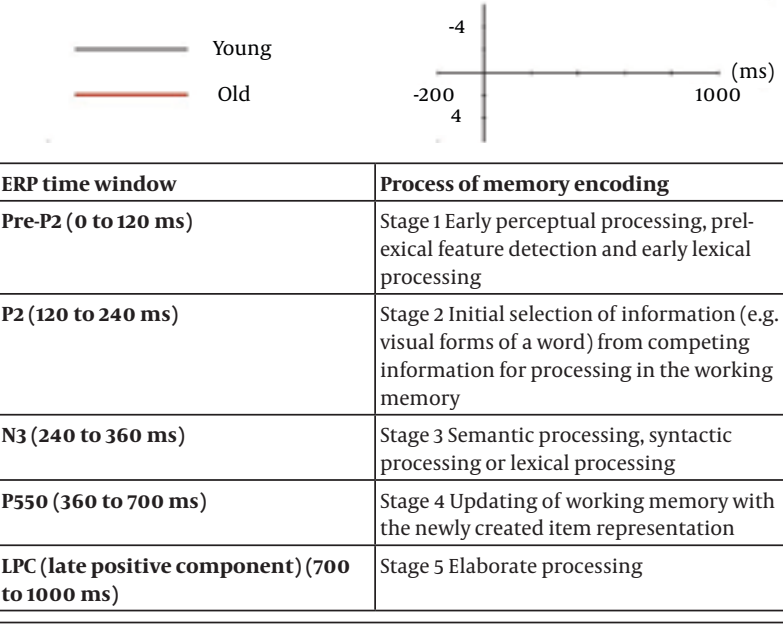

It is illustrated by a selection of two subtracted waveforms of the eventrelated potentials (ERP) (F3 and F7), elicited by correctly identified and $\underline{\text { missed trials in perceptual condition of the younger and older groups (9). }}$ of P550 and LPC to elaborative processing. Semanticallylearnt characters elicited LPC with significantly greater amplitudes over the frontal pole regions. The significant SME in the frontal regions of LPC in both conditions suggested that coordination and incorporation of the orthographic information to mental representation were essential to both task conditions. Underlying processes expressed by frontal pole SME, as shown in the semantic condition, might be important for strategic operations that can further enhance encoding. Differences in SMEs N3 between conditions were also significant.

Kuo and Liu(9) identified that younger adults performed better than older adults in both conditions. Semantic orientation helped older adults to perform better in a perceptual task (1.17 \pm 0.73 vs. $0.63 \pm 0.4, P=0.01)$. Significant SMEs were found in $\mathrm{P} 2$ and $\mathrm{N} 3$ windows in both conditions and P550 window in the semantic condition in older adults (Figure 1). Correlation analysis on LPC indicated positive correlation between SME amplitude and behavioral measures in the perceptual condition. When semantic processing was required, ERP differences in earlier windows (P2, $\mathrm{N} 3$, and $\mathrm{P} 550$ ) were less evident between the younger and old groups. It was not till the LPC window, in which more definite age-related differences indicated by larger frontal and central SMEs in the younger sample were found. In the perceptual condition, however, differences were already shown at P550 window, suggesting that older people might have more difficulty performing perceptual processing to result in successful encoding. The older group's difficulty in perceptual encoding might be due to a failure to update and maintain memory representation. Age-related decline in encoding may be due in part to changes in the frontal lobe function.

\subsection{Encoding Processes in Young Adults}

The results of our two studies with a review of previous literature showed that encoding processes in younger adults could be broken down to five stages (Figure 2); stage one (pre-P2): early perceptual processing, prelexical feature detection, and early lexical processing $(13,14)$; stage two (P2): initial selection of information (e.g. visual forms of a word) from competing information for processing in the working memory (15-18); stage three (N3): semantic, syntactic, or lexical processing $(16,19,20)$; stage four (P550): updating of working memory with the newly created item representation $(16,21,22)$; and stage five (LPC): elaborate processing $(15,23,24)$.

Except for the elaborate processing in stage five, other processes occur unconsciously (16). In general, these stages can be imagined to go sequentially. Both perceptual and semantic encodings would involve all these processes. However, there are some differences between these two kinds of encodings.

In the investigation by Kuo and Liu (7), a difference was found in a stage-two process, associated with attention and higher-order perceptual processing, namely analysis of visual characteristics $(16,25,26)$. This process has also 
been associated with top-down matching process of comparing sensory inputs with mental representations, happening in working memory (27). More activities in the parietal regions were associated with perceptually learned Chinese characters. This suggested that during perceptual learning, processing of perceptual rather than semantic features was more readily elicited. This was likely to be due to greater attentional resources needed for inspection of the characters' appearances.

Another finding that differentiates perceptual from semantic processing is identification of more activities in the left inferior region of the brain in the stage three of the encoding process. In the previous studies, functionalities of these activities were not clearly elucidated. At this stage, they have been ascribed to accessing semantic memory, elicited by responding to meaningful or potentially meaningful stimuli (28). A transient implicit binding process of conceptual representation for processing also occurs at this stage (28). Therefore, semantic encoding may be associated with more efficient processing of meaning and initiating access to semantic memory. In stage five of the process, semantic encoding consistently involves activities in the frontocentral regions of the brain (12, 24, 29). In Cansino and Trejo-Morales study (12), participants were asked to encode pictures of animals, while in Otten and Rugg's (29) words were encoded. Weyerts et al. (24) used a paired-associate learning paradigm with varied relatedness of word pairs. The activities were more than those elicited by perceptual encoding, so the contribution by the frontal regions might be specific to semantic encoding (7). The underlying processes revealed by the frontal activities further enhanced memory. These could be related to the strategic processes brought about by the executive functions which help to elaborate information and integrate mental representation with long-term memory (7).

\subsection{Encoding Processes in Old Adults}

Previous investigations have suggested that older adults might experience significant deficits during encoding $(30,31)$. These deficits can be attributed to the differences in brain activities, when compared to younger adults. Overall, older adults are able to process information both perceptually and semantically. In addition, they seem to have more difficulties with perceptual type of encoding (9) and stage five of the encoding process.

Little differences have been found in stages one and two of the encoding process $(9,10,26)$. The results from these investigations showed insignificant between-group dif ferences. This indicated that older and younger adults might undergo similar early visual analyses of the input information. Kuo and Liu (9) instructed younger and older adults to encode a list of Chinese characters perceptually and semantically. Differences in the activities during stage three were identified. The differences indicated that older adults' implicit binding processes (stage three) may be less efficient than those of younger adults. Little or no differences have been identified in stage four of the encoding process between younger and older adults $(9,10,26,32)$. While this indicates that the updating process is similar in both younger and older adults, there seems to be material-specific (verbal vs. nonverbal) difference when information is processed perceptually at this stage. Friedman, Ritter and Snodgrass (33) and Kuo and Liu (9) asked older adults to encode verbal materials and both demonstrated that older adults showed less brain activities. The results suggested that older adults may experience difficulties in updating the perceptual representation of the characters. This would subsequently affect maintenance of the representation in working memory and incorporation of the learned characters into long-term memory for retrieval. However, results from Gutchess, Ieuji and Federmeier (34) who asked older adults to encode nonverbal (scenic pictures) materials indicated otherwise. Their results were more commonly associated with processing of semantic information (10, 16). In line with this, other studies have suggested that pictures could be processed more spontaneously using semantically- rather than perceptually-based strategies (35-37). Therefore, results of Gutchess, Ieuji (34) may only be applied to pictures as the encoding material.

Most consistent age differences come from the stage five (elaborate processing) of the encoding process. At this stage, links are created between the new information and prior knowledge in long-term memory. Older adults tend not to encode information with much elaboration. This seems to be true in both perceptual (34) and semantic encoding conditions (32), indicating that encoding may be affected by aging indiscriminately across tasks. Other results suggested that older adults might find it more challenging to maintain the information for further elaboration in working memory than their younger counterparts $(9,10)$. Friedman and Trott (32) observed laterality differences among younger and older adults in the frontal brain region. Activities from the frontal region might be associated with integration and coordination of the processed information, which are functions of the frontal lobes $(7,10)$. Therefore, difficulty with maintaining information in older adults might be partly due to changes in frontal lobe function.

\subsection{Important Structures Serving Encoding}

Neuroimaging methods such as functional magnetic resonance imaging have been used to localize structures involved in encoding. Perceptual encoding appears to be modulated by sensory cortices, in which stimuli are initially perceived and processed. For visual information, the parietal and occipital cortices (38) and fusiform gyrus $(39,40)$, and for auditory stimuli, the superior temporal gyrus may be involved (41). As working memory is often involved, parietal lobes have been identified as important substrates $(42,43)$. Particularly, spatial work- 
ing memory tasks may involve the right hemisphere to a greater extent, while verbal and object working memory tasks may rely more on the left hemisphere $(44,45)$.

According to the hemispheric encoding/retrieval asymmetry (46) model, semantic encoding involves the left prefrontal cortex (PFC) (47). In addition to the left PFC, temporal regions are also commonly activated (48) by both verbal and nonverbal stimuli. Depending on the type of knowledge demanded (eg, color, tool, animal or motion), other associated brain areas are also activated. Since PFC is involved in various tasks which need executive functions (49), its role may be attention, manipulation and strategic operation of working memory $(50,51)$, linking new information with information from long-term memory, regulating input to the medial temporal lobe (MTL) structures, and modulating activity in the sensory neocortex (52).

In addition, perceptual and semantic encodings typically involve MTL, especially the hippocampus (53). MTL can be activated by both verbal and nonverbal stimuli. In studies using verbal stimuli, MTL activation has been more left lateralized, and in studies using nonverbal stimuli, it has been more bilaterally activated. MTL structures interact with other brain regions for material-specific processing: parietal lobes are allocated for spatial processing and temporal lobes for object feature processing (54).

\subsection{Aging and Brain Activation Changes}

Neural changes associated with aging, mostly deteriorations, are well documented (55). These changes include declines in hippocampus and frontal lobe volumes, shrinkage in white matter and its tracts, and reduction of dopamine producing ability. Frontal lobe and MTL areas that are important for memory encoding are often affected (56).

Grady, McIntosh, Horwitz, Maisog, Ungerleider, Mentis (57) examined face encoding and found that younger adults showed activations in the anterior cingulate, PFC and left temporal cortex. In contrast, significant activations in the inferior frontal cortex and MTL were not present during encoding in older adults. This result has been replicated in other studies. Anderson, Iidaka, Cabeza, Kapur, McIntosh and Craik (58) used word pairs in a paired-associate learning paradigm and found that left inferior activation was significant only in younger adults. However, older adults showed higher activation compared to younger adults in inferior parietal cortexes. The results from Anderson and Iidaka study (58) may indicate that older adults had a tendency to rely more on shallow perceptual encoding. Activation of the left inferior PFC in younger adults was associated with semantic encoding $(59,60)$. Reduced activation of left inferior PFC regions has also been found by Stebbins, Carrillo, Dorfman, Dirksen, Desmond and Turner (61). They required their participants to make semantic/nonsemantic judgments about words during encoding.

Differences in activation of the left inferior PFC would decrease if both age groups were instructed to perform a semantic task. Logan, Sanders, Snyder, Morris and Buckner (62) showed that inducing older adults to engage in effective encoding strategies resulted in activation of left PFC to the level of younger adults. However, there still seemed to be differences in patterns of activation in such instances. Specifically, frontal activations were left lateralized in the younger group, but located bilaterally in the older group (31). Bilateral activation could be considered as a compensatory measure. Older adults over-recruit to compensate for loss of memory encoding process or its inefficiency. In Morcom's study, difference was found in the left anterior temporal cortex, which was activated only in younger adults. Overall, left PFC appears to play an important role in older adults' memory functions.

\section{Conclusions}

This paper reviewed unique processes of memory encoding. Younger and older adults shared similar early perceptual processing and selection of information. However, they showed differences in sematic processing, updating of working memory, and elaborate processing. Such differences could be accounted by the declines in hippocampus and frontal lobes volumes and functions, shrinkage in white matter and its tracts, and reduction in abilities for producing dopamine in an aging brain.

\section{Financial Disclosure}

There was no conflict of interest.

\section{Funding/Support}

The work was supported in part by the School of Science and Health Research Seed Grant from the University of Western Sydney, Australia, awarded to Karen P.Y. Liu.

\section{References}

1. Atkinson RC, Shiffrin RM. The Control of Short-Term Memory. SCI AM.1971;225(2):82-90.

2. Craik FI, Rose NS. Memory encoding and aging: a neurocognitive perspective. Neurosci Biobehav Rev. 2012;36(7):1729-39.

3. Baddeley AD, Eysenck MW, Anderson MC. Memory.London: Psychology Press; 2009.

4. Seifert T. Human learning and motivation: Readings. 1st ed: John's Memorial University; 1995.

5. Craik FIM, Lockhart RS. Levels of processing: A framework for memory research. J VERB LEARN VERB BE. 1972;11(6):671-84.

6. Wu JKY, Liu KPY, Chan CCH. Memory strategies used for recall task. Neurorehabil Neural Repair. 2006;20:91.

7. Kuo MC, Liu KP, Ting KH, Chan CC. Differentiation of perceptual and semantic subsequent memory effects using an orthographic paradigm. Brain Res. 2012;1486:82-91.

8. Chase WG, Simon HA. The mind's eye in chess. In: Chase WG editor. Visual information processing: proceedings.. New York: Academic Press; 1973.

9. Kuo MC, Liu KP, Ting KH, Chan CC. Age-related effects on perceptual and semantic encoding in memory. Neuroscience. 2014;261:95-106.

10. Cansino S, Trejo-Morales P, Hernandez-Ramos E. Age-related changes in neural activity during source memory encoding in young, middle-aged and elderly adults. Neuropsychologia. 2010;48(9):2537-49.

11. Wagner AD, Koutstaal W, Schacter DL. When encoding yields re- 
membering: insights from event-related neuroimaging. Philos Trans R Soc Lond B Biol Sci.1999;354(1387):1307-24

12. Cansino S, Trejo-Morales P. Neurophysiology of successful encoding and retrieval of source memory. Cogn Affect Behav Neurosci. 2008;8(1):85-98.

13. Compton PE, Grossenbacher P, Posner MI, Tucker DM. A cognitive-anatomical approach to attention in lexical access. J Cogn Neurosci.1991;3(4):304-12.

14. Sereno SC, Rayner K, Posner MI. Establishing a time-line of word recognition: evidence from eye movements and event-related potentials. Neuroreport.1998;9(10):2195-200.

15. Bridger EK, Wilding EL. Requirements at retrieval modulate subsequent memory effects: An event-related potential study. Cogn Neurosci. 2010;1(4):254-60.

16. Mangels JA, Picton TW, Craik FIM. Attention and successful episodic encoding: an event-related potential study. Cognitive Brain Research. 2001;11(1):77-95.

17. Otten LJ, Sveen J, Quayle AH. Distinct patterns of neural activity during memory formation of nonwords versus words. J Cogn Neurosci. 2007;19(11):1776-89.

18. Posner MI, Rothbart MK. Attention, self-regulation and consciousness. Philos Trans R Soc Lond B Biol Sci.1998;353(1377):1915-27.

19. Grunwald T, Lehnertz K, Heinze HJ, Helmstaedter C, Elger CE. Verbal novelty detection within the human hippocampus proper. Proc Natl Acad Sci U S A. 1998;95(6):3193-7.

20. Kutas M. Views on how the electrical activity that the brain generates reflects the functions of different language structures. Psychophysiology. 1997;34(4):383-98.

21. Donchin E, Coles MGH. Is the P300 component a manifestation of context updating? BEHAV BRAIN SCI. 2010;11(3):357.

22. Picton TW. The P300 wave of the human event-related potential J Clin Neurophysiol. 1992;9(4):456-79.

23. Kuo MCC. Factors involved in memory encoding and their implications for the memory performance of older adults and people with mild cognitive impairment. World J Neuro. 2012;2(2):103-12.

24. Weyerts H, Tendolkar I, Smid HG, Heinze HJ. ERPs to encoding and recognition in two different inter-item association tasks. Neuroreport. 1997;8(7):1583-8.

25. Smith ME. Neurophysiological Manifestations of Recollective Experience during Recognition Memory Judgments. J Cogn Neurosci.1993;5(1):1-13.

26. Tellez-Alanis B, Cansino S. Incidental and intentional encoding in young and elderly adults. NeuroReport. 2004;15(11):1819-23.

27. Freunberger R, Klimesch W, Doppelmayr M, Holler Y. Visual P2 component is related to theta phase-locking. Neurosci Lett. 2007;426(3):181-6

28. Kutas M, Federmeier KD. Thirty years and counting: finding meaning in the $\mathrm{N} 400$ component of the event-related brain potential (ERP). Annu Rev Psychol. 2011;62:621-47.

29. Otten LJ, Rugg MD. Electrophysiological correlates of memory encoding are task-dependent. COGNITIVE BRAIN RES. 2001;12(1):11-8

30. Gutchess AH, Welsh RC, Hedden T, Bangert A, Minear M, Liu LL, et al. Aging and the neural correlates of successful picture encoding: frontal activations compensate for decreased medial-temporal activity. J Cogn Neurosci. 2005;17(1):84-96.

31. Morcom AM, Good CD, Frackowiak RS, Rugg MD. Age effects on the neural correlates of successful memory encoding. Brain. 2003;126(Pt 1):213-29.

32. Friedman D, Trott C. An event-related potential study of encoding in young and older adults. NEUROPSYCHOLOGIA. 2000;38(5):542-57.

33. Friedman D, Ritter W, Snodgrass JG. ERPs during study as a function of subsequent direct and indirect memory testing in young and old adults. COGNITIVE BRAIN RES.1996;4:1-13.

34. Gutchess AH, Ieuji Y, Federmeier KD. Event-related potentials reveal age differences in the encoding and recognition of scenes. Cogn Neurosci. 2007;19(7):1089-103.

35. Paivio A. Imagery and Verbal Processes.: Taylor \& Francis; 1971.

36. Mintzer MZ, Snodgrass JG. The Picture Superiority Effect: Support for the Distinctiveness Model. AM J PSYCHOL.1999;112(1):113.

37. Levie WH. Research on pictures: A guide to the literature. In: Willows DM, Houghton HA editors. The Psychology of Illustration: Volume 1 Basic Research.. New york: Springer-Verlag;1987. pp. 1-50.

38. Blaxton TA. Combining disruption and activation techniques to map conceptual and perceptual memory processes in the human brain. In: Foster JK, Jelicic M editors. Memory: Systems, Process, O Function?. Oxford: Oxford University Press;1999. pp. 104-29.

39. Cavina-Pratesi C, Kentridge RW, Heywood CA, Milner AD. Separate channels for processing form, texture, and color: evidence from FMRI adaptation and visual object agnosia. Cereb Cortex. 2010;20(10):2319-32.

40. Cavina-Pratesi C, Kentridge RW, Heywood CA, Milner AD. Separate processing of texture and form in the ventral stream: evidence from FMRI and visual agnosia. Cereb Cortex. 2010;20(2):433-46.

41. Wagner AD, Bunge SA, Badre D. Cognitive control, semantic memory, and priming: Contributions from prefrontal cortex. 3rd edCambridge, Massachusetts: MIT Press; 2004.

42. Honey GD, Fu CH, Kim J, Brammer MJ, Croudace TJ, Suckling J, et al. Effects of verbal working memory load on corticocortical connectivity modeled by path analysis of functional magnetic resonance imaging data. Neuroimage. 2002;17(2):573-82.

43. Owen AM. The functional organization of working memory processes within human lateral frontal cortex: the contribution of functional neuroimaging. Eur J Neurosci.1997;9(7):1329-39.

44. Smith EE. Storage and Executive Processes in the Frontal Lobes SCI.1999;283(5408):1657-61.

45. Smith EE, Jonides J, Marshuetz C, Koeppe RA. Components of verbal working memory: Evidence from neuroimaging. PNAS 1998;95(3):876-82.

46. Tulving E, Kapur S, Craik FI, Moscovitch M, Houle S. Hemispheric encoding/retrieval asymmetry in episodic memory: positron emission tomography findings. Proc Natl Acad Sci U S A. 1994;91(6):2016-20.

47. Prince SE, Tsukiura T, Cabeza R. Distinguishing the neural correlates of episodic memory encoding and semantic memory retrieval. Psychol Sci. 2007;18(2):144-51.

48. Martin A. Functional neuroimaging of semantic memory. In: Cabeza R, Kingstone A editors. Handbook of Functional Neuroimaging of Cognition.. Cambridge: MIT Press; 2001. pp.153-86.

49. Kane MJ, Engle RW. The role of prefrontal cortex in workingmemory capacity, executive attention, and general fluid inelligence: An individual-difference perspective. Psychonomic Bulletin \& Review. 2002;9:637-71

50. Curtis CE, D'Esposito M. Persistent activity in the prefrontal cortex during working memory. TRENDS COGN SCI. 2003;7(9):415-23.

51. Postle BR. Working memory as an emergent property of the mind and brain. Neuroscience. 2006;139(1):23-38.

52. Summerfield C, Greene M, Wager T, Egner T, Hirsch J, Mangels J Neocortical connectivity during episodic memory formation. PLoS Biol. 2006;4(5).

53. Tulving E. Concepts of memory. In: Tulving E, Craik FIM editors The Oxford Handbook of Memory. New York: Oxford University Press; 2000. pp. 33-43.

54. Köhler S, Moscovitch M, Winocur G, Houle S, McIntosh AR. Networks of domain-specific and general regions involved in episodic memory for spatial location and object identity. NEUROPSYCHOLOGIA. 1998;36(2):129-42.

55. Raz N, Lindenberger U, Rodrigue KM, Kennedy KM, Head D, Williamson A, et al. Regional brain changes in aging healthy adults general trends, individual differences and modifiers. Cereb Cortex. 2005;15(11):1676-89.

56. Giorgio A, Santelli L, Tomassini V, Bosnell R, Smith S, De Stefano $\mathrm{N}$, et al. Age-related changes in grey and white matter structure throughout adulthood. Neuroimage. 2010;51(3):943-51.

57. Grady CL, McIntosh AR, Horwitz B, Maisog JM, Ungerleider LG Mentis MJ, et al. Age-related reductions in human recognition memory due to impaired encoding. Science.1995;269(5221):218-21.

58. Anderson ND, Iidaka T, Cabeza R, Kapur S, McIntosh AR, Craik FI The effects of divided attention on encoding- and retrieval-related brain activity: A PET study of younger and older adults. J Cogn Neurosci. 2000;12(5):775-92.

59. Buckner RL, Kelley WM, Petersen SE. Frontal cortex contributes to human memory formation. Nat Neurosci.1999;2(4):311-4

60. Kapur S, Craik FI, Tulving E, Wilson AA, Houle S, Brown GM. Neuroanatomical correlates of encoding in episodic memory: levels of processing effect. Proc Natl Acad Sci U S A 1994;91(6):2008-11. 


\section{Kuo MCC et al.}

61. Stebbins GT, Carrillo MC, Dorfman J, Dirksen C, Desmond JE, Turner DA, et al. Aging effects on memory encoding in the frontal lobes. Psychol Aging. 2002;17(1):44-55.
62. Logan JM, Sanders AL, Snyder AZ, Morris JC, Buckner RL. Under-Recruitment and Nonselective Recruitment. Neuro. 2002;33(5):827-40. 